Horizons philosophiques

\title{
L'état idéal de finitude chez Gadamer
}

\section{Alain Beaulieu}

Volume 7, numéro 2, printemps 1997

L’héritage de l’herméneutique

URI : https://id.erudit.org/iderudit/801045ar

DOI : https://doi.org/10.7202/801045ar

Aller au sommaire du numéro

Éditeur(s)

Collège Édouard-Montpetit

ISSN

1181-9227 (imprimé)

1920-2954 (numérique)

Découvrir la revue

Citer cet article

Beaulieu, A. (1997). L'état idéal de finitude chez Gadamer. Horizons

philosophiques, 7(2), 75-90. https://doi.org/10.7202/801045ar d'utilisation que vous pouvez consulter en ligne.

https://apropos.erudit.org/fr/usagers/politique-dutilisation/ 


\section{L'ÉTAT IDÉAL DE FINITUDE CHEZ GADAMER}

La philosophique herméneutique défendue par Gadamer dans Vérité et méthode mobilise un état de finitude que nous qualifions d'idéal. Le choix de cet adjectif pour caractériser la finitude selon Gadamer s'autorise du fait que l'herméneutique philosophique accorde systématiquement, comme nous le verrons, valeur de connaissance et de sens à la relativité des occurrences de compréhension. On constate alors que la compréhension par rapport à laquelle Gadamer définit la finitude n'est pas totalement externe et autonome par rapport à elle et conserve pour la finitude un rôle normatif. Nous opposons à cet état idéal de finitude un état non-idéal qui apparaît à la modernité et pour lequel l'incarnation d'un monde intelligible et normatif n'a plus lieu. Gadamer s'est volontairement fermé à ce type de finitude en rejetant la possibilité que la finitude de la compréhension puisse reposer sur l'absence de lois. L'herméneutique philosophique récupère donc à ses frais la conception traditionnelle de la finitude en tentant de décrire un monde où la bonne circulation du sens vient assurer l'avenir grandissant de la vérité à l'intérieur des occurrences de compréhension.

Nous voudrions dans un premier temps (1) montrer comment la dynamique générale de la bonne circulation du sens est reprise dans le déploiement interne des trois sections de Vérité et méthode. On tentera ensuite (2) de faire ressortir la valeur néoclassique de Vérité et méthode en établissant un parallèle entre le genre de finitude propre à l'herméneutique philosophique et une étude de Winckelmann sur les statues grecques. On constatera qu'une nostalgie de la Grèce colore Vérité et méthode et amène l'herméneutique philosophique à récupérer de l'Antiquité la définition de la finitude comme manque vis-à-vis de la plénitude. En s'en tenant à cette conception de la finitude, nous voudrions par la suite indiquer (3) comment Gadamer écarte de son chemin le point de vue moderne 
sur la finitude dont nous verrons la première manifestation dans l'analyse kantienne du jugement réfléchissant. Finalement (4), nous nous demanderons si l'herméneutique philosophique qui récupère une notion traditionnelle de finitude ne constitue pas un recul par rapport aux avancées de projets tels que ceux du second Heidegger et de Derrida. En outre, nous supposerons qu'une confrontation productive entre les pensées de Gadamer, du deuxième Heidegger et de Derrida pourra avoir lieu sur les bases de la reconnaissance des deux types de finitude identifiés. Cette rencontre devrait ouvrir sur de nouvelles possibilités pour herméneutique jusqu'à, peut-être, en signaler sa fin.

\section{La finitude dans Vérité et méthode}

La série de réflexions théoriques sur les phénomènes de l'interprétation et de la compréhension exposées dans Vérité et méthode par Gadamer, comme on le sait, vise à dégager le concept de vérité de l'idée de méthode. Pour ce faire, et tel qu'exposé dans la première partie de Vérité et méthode, Gadamer souhaite d'abord contrer le mouvement de subjectivation propre à la modernité en refusant l'opposition établie par Kant entre connaissance théorético-objective et jugement subjectif du goût. L'union de ces deux termes a pour conséquence d'accorder une valeur cognitive à la sphère du jugement esthétique. Gadamer reconnaît ainsi en l'art un lieu de médiation de la vérité1.

L'herméneutique gadamérienne franchit un pas de plus dans cette direction en thématisant les notions de jeu ${ }^{2}$ et de «non-différenciation esthétique» 3 d'après lesquelles toute image référerait de façon ontologique à son modèle. Gadamer écrit : "son être véritable [de l'œuvre d'art] est inséparable de sa représentation et cependant la représentation procède de

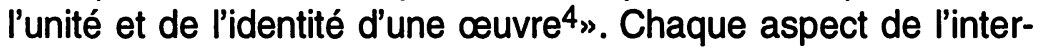
prétation de l'œuvre d'art nous livre donc l'œuvre d'art ellemême.

1. Hans-Georg Gadamer, Vérité et méthode, Paris, Seuil, 1996, p. 15.

2. Ibid., p. 119.

3. Ibid., p. 135.

4. Ibid., p. 140. 
Dans le même esprit, la seconde section de Vérité et méthode souhaite redonner une valeur positive aux préjugés pour rendre justice au caractère historiquement fini de l'être humain ${ }^{5}$. Ceci en considérant les préjugés comme étant d'emblée constitutifs de tout phénomène de compréhension. Celle-ci n'est donc plus reproduction d'un sens origine/ 6 mais produit plutôt du sens nouveau au même rythme où les préjugés sont mis à l'épreuve ${ }^{7}$. L'émergence perpétuelle de sens se produit dans un processus de fusion continuel et mouvant des horizons passé et présent ${ }^{8}$ à partir duquel naissent les possibilités historiques de compréhension qui, bien qu'à chaque fois différentes, n'en participent pas moins de la vérité elle-même en la révélant.

La perpétuelle fusion des horizons appellerait une incessante et universelle quête langagière thématisée dans la troisième section de Vérité et méthode. Le langage qui vulgarise les "bons préjugés" communie chaque fois avec la chose elle-même bien que l'accomplissement de l'idéal d'objectivation total et de compréhension universelle du monde soit reporté à l'infini. Gadamer écrit : "Ce qu'est le monde lui-même ne diffère en rien des aspects sous lesquels il s'offre ${ }^{9}$ ». Ceci parce que les mots, bien que n'ayant jamais de significations transcendantales hors du monde, constituent notre seul mode d'accès au monde. Qui plus est, notre accès ultimement langagier au monde serait en mesure de rendre les mots égaux aux choses. Gadamer écrit : "Le monde linguistique propre dans lequel on vit n'est pas une barrière qui empêche d'accéder à la connaissance de "l'être en soi"10".

Comme on le constate, l'herméneutique philosophique de Gadamer accorde aux occurrences particulières et relatives des expériences esthétique, historique et langagière, le pouvoir de révéler l'être lui-même. C'est ainsi que la non-différenciation esthétique en vient à dissoudre les hiatus entre l'image et le
5. Ibid., p. 290.
6. Ibid., p. 318.
7. Ibid., p. 328.
8. Ibid., p. 328.
9. Ibid., p. 472.
10. Ibid., p. 471. 
modèle, la fusion des horizons, à assimiler passé et présent et l'usage du langage, à annihiler l'espace entre les choses et les mots.

Suite à ces analyses, nous qualifions d'idéal l'état de finitude mobilisé par l'herméneutique de Gadamer. Cet état est idéalement fini parce qu'on lui garantit systématiquement la possibilité d'une bonne circulation du sens dans l'histoire en l'adaptant chaque fois aux différentes situations auxquelles la finitude doit se confronter.

Cette jonction entre le «relatif» et "l'absolu» est fondamentale à l'intelligence de l'herméneutique gadamérienne. La justification de cette séduisante fusion mise en œuvre dans Vérité et méthode est exposée dans un petit texte qui lui est postérieur et qui s'intitule «philosophie et poésie». Gadamer y écrit, dans un contexte où il parle des dires poétique et philosophique et pour lequel nous retiendrons surtout ce qui est dit du second :

Les deux manières de parler, la manière poétique et la manière philosophique, se partagent ainsi un trait commun. Elles ne peuvent être "fausses". Car il n'y a pas d'étalon de mesure externe à elles qui leur serait applicable et auquel elles pourraient correspondre. Elles sont pourtant tout autre chose que fortuites. Elles courent un risque d'un genre tout particulier : elles peuvent se manquer elles-mêmes. Dans les deux cas, on ne constaterait pas alors qu'elles ne correspondent pas aux choses, on remarquerait seulement que la parole $y$ devient "vide». Dans le cas de la poésie, cela signifie qu'au lieu de résonner pour elle-même, elle ne fait entendre que l'écho, soit d'une autre poésie, soit de la rhétorique de la vie courante. Dans le cas de la philosophie, on remarque alors que le discours philosophique reste empêtré dans une argumentation purement formelle, ou encore qu'il sombre dans des sophismes vides $^{11}$.

11. Hans-Georg Gadamer, L'actualité du beau, Aix-en-Provence, Alinéa, 1992, p. 186. Avant Gadamer, Heidegger avait déjà déclaré caduque la distinction entre le relatif et l'absolu. Voir Martin HEIDEGGER, Les problèmes fondamentaux de la phénoménologie, Paris, Gallimard, 1985, p.266-267 où il est écrit : "Poser des vérités éternelles demeure une affirmation fantaisiste, de même que cela demeure une mécompréhension naĩve que de croire que la vérité est livrée au relativisme." 
Tout étalon de mesure étant lui-même fini, l'énoncé philosophique n'est ni relatif, ni absolu, mais toujours «vrai»; il est finitude idéale. Ni fausse, ni fortuite, la bonne parole philosophique ne peut donc qu'atteindre la vérité dans la singularité de son dire. Accordant pleine confiance au dialogue socratique que nous sommes et idéalisant l'état de finitude de l'homme, Gadamer renoue avec l'Antiquité grecque et un optimisme dialectique sans bornes en fuyant la possibilité qu'une finitude soit inapte à réaliser l'accomplissement ou la transmission du sens.

Dans la prochaine section, nous exemplifierons l'amour de Gadamer pour la Grèce antique en établissant un parallèle entre la finitude comprise par l'herméneutique et la sculpture grecque. Nous constaterons dans les deux cas qu'il y a alliance parfaite de la matière sensible et de la forme idéale. Dans la troisième section de notre travail, nous questionnerons la légitimité de la reprise par Gadamer de la vision grecque de la finitude humaine au détriment d'une conception plus moderne de la finitude. Mais d'abord, les statues grecques.

\section{Herméneutique et sculpture grecque}

Tel que nous l'avons vu, l'herméneutique de Gadamer comprend l'advenir de l'être dans la relativité de ses expériences. Cette intégration l'autorise à définir une ontologie à l'intérieur de laquelle le caractère circonstantiel de la compréhension a la capacité de manifester l'en-soi'12. En ce sens, Gadamer écrit :

Toute appropriation de la tradition est historiquement différente - ce qui ne veut pas dire qu'elle n'en soit qu'une conception obscurcie; chacune d'elle est bien au contraire l'expérience d'un "aspect» de la chose même13.

D'où l'idée sous-jacente au projet gadamérien d'un absolu temporellement déployé et compris au gré de notre propre finitude. Celle-ci ne devenant plus une entrave absolue à notre compréhension de l'être langagier mais sa condition de possibilité.

12. Gadamer, Vérité et méthode, Paris, p. 500.

13. Ibid., p. 498. 
Cet état herméneutique semble se situer assez près de l'expression idéale de la réalité et du monde atteint par les sommets de la sculpture de la Grèce antique. Le grand intérêt porté par Gadamer aux humanités et à l'art semble bien se conjuguer avec la supposition d'une intime fascination pour les statues grecques. Leur charme a bien pu lui inspirer secrètement sa conception herméneutique de la finitude. Notre hypothèse de travail est en fait que l'état idéal de finitude thématisé par l'herméneutique de Gadamer est taillé dans la même pierre qui a servi aux sculpteurs du grand art de la Grèce antique pour réaliser leurs idéaux de beauté.

La nostalgie de Gadamer pour cette grande période incite la pensée herméneutique à y revenir en se modelant sur elle. Sans l'avouer explicitement, Gadamer semble nous dire que la pensée ne peut s'éloigner de la Grèce sans s'altérer et que l'unique moyen pour nous de devenir grands, c'est d'imiter les anciens.

Pour illustrer cette exigence d'un retour au mode de pensée grec, nous comparerons l'idéalité de la pensée de Gadamer et celle de la sculpture grecque décrite par Winckelmann. Celui-ci présente la statue grecque en décrivant ses «contours grands et virils que les maîtres grecs ont donnés à leurs statues, sans bouffissure et sans empâtement superflu [et conçues] de manière à ne pas imposer la moindre contrainte à l'action de la nature ${ }^{14}$ ». Représentant par ses formes le sens de la mesure, de la pondération, la perfection de la chair sans irrégularités et la jeunesse des divinités, les chefs-d'œuvre grecs se distinguent par une noble simplicité et une grandeur sereine, aussi bien dans l'attitude que dans l'expression. De même que les profondeurs de la mer restent calmes en tous temps, quelque furieuse que soit la surface, de même l'expression, dans les figures des Grecs, montre, même au sein des passions, une âme grande et toujours égale ${ }^{15}$.

De la même façon que la statue grecque réalise l'union idéale du sensible et du supra-sensible, l'état de finitude propre à l'herméneutique gadamérienne réussit à joindre la proposition

14. J.-J. Winckelmann, Réflexions sur l'imitation des œuvres grecques en peinture et en sculpture, Paris, Aubier, 1990, p. 103.

15. Ibid., p. 143. 
philosophique et la vérité en garantissant la possibilité d'une bonne compréhension, transmission du sens et adéquation des mots à «l'en-soi». Le tandem "matière et forme" de la statue grecque se nomme maintenant «langage et être» chez Gadamer. Dans les deux cas, on souhaite intégrer les termes l'un dans l'autre jusqu'à en rendre indistinct leur caractère relatif ou absolu. Dans les deux cas les normes de l'infini s'incarnent dans le fini.

Cette vaste entreprise d'idéalisation léguée à l'Occident par la culture grecque peut à elle seule expliquer l'effectivité de la philosophie. Elle prend maintenant forme dans l'herméneutique de la finitude idéale de Gadamer. Ce retour au modèle d'idéalité grecque nous amène à nous demander, un peu dans le même esprit où Gadamer a qualifié Bergson de «descendant tardif de la critique romantique de la pensée mécaniste ${ }^{16}$ ", si son herméneutique ne constitue pas en fait les restes du néoclassicisme. Bien sûr les statues grecques nous parlent encore. Mais que nous disent-elles sinon qu'elles appartiennent à un autre monde mystificateur et surnaturel?

Sous son mot d'ordre «retour à Platon», à la positivité du dialogue que nous sommes et qui chemine dans le dire de l'être, dans son éloge déguisé de l'idéalité de la statue grecque, Gadamer oublie de prendre en compte la possibilité d'une finitude qui soit radicalement imparfaite, d'une finitude qui ne soit plus "sans bouffissure et sans empâtement". Nous qualifions ce type de finitude qui échappe à Gadamer et qui est apparu avec la modernité, de non-idéal.

Nous tenterons de montrer comment ce modèle de finitude a été pris en charge par les pensées du second Heidegger et de Derrida. Mais d'abord, nous essayerons de voir de quelle façon Kant, par son analyse du jugement réfléchissant, inaugure ce nouveau type de finitude auquel ne fait face aucune transcendance idéale. Ce nouvel état fini consacré par Kant a été explicitement écarté comme possibilité et rendu illégitime par Gadamer dans le geste inaugural de Vérité et méthode.

16. Gadamer, Vérité et méthode, p. 263. 


\section{Le jugement réfléchissant}

Platon savait que les Dieux n'avaient pas besoin de philosophie et que cette exigence était l'affaire des mortels. De façon similaire, Gadamer affirme que la finitude historique de notre être impose des limites à l'universalité de la compréhension; la finitude se définissant chez Gadamer par contraste avec la compréhension universelle ${ }^{17}$. Cette dissemblance entre finitude et compréhension universelle reste cependant incomplète en ce que cette dernière demeure normative du savoir pour la finitude. Gadamer sait que l'universalité de la compréhension appartient à un autre monde impossible à atteindre absolument. Néanmoins, cette "entité supra-sensible" constitue un modèle imitable et à imiter dans et par la finitude. Ainsi les hommes ne possèdent ni ne posséderont jamais l'omniscience des Dieux pas plus que la compréhension universelle du monde tout en pouvant espérer cheminer vers une vérité grandissante par l'effort dialogique. Voilà le sens classique accordé à la finitude visà-vis d'un infini transcendant et normatif pour la finitude tel que repris par l'herméneutique philosophique de Gadamer.

Cet état idéal de finitude comme manque par rapport à un infini normatif demeure cependant partiel face à un autre genre de finitude. II s'agit d'une finitude pour laquelle la sphère universelle distributrice de normes fait défaut. C'est cette situation à laquelle s'est confronté Kant dans son analyse du jugement réfléchissant ${ }^{18}$.

Dans la troisième critique lorsqu'il est question du jugement réfléchissant, Kant pense une situation où l'universel n'est plus

17. Ibid., p. 252. Voir aussi formulation semblable p. 138. II est important de noter ici que l'universalité gadamérienne discutée à partir de maintenant dans notre travail sera toujours celle qui est comprise dans l'expression d'une compréhension universelle que Gadamer veut opposer à la finitude et non pas l'universalité de l'herméneutique thématisée à la toute fin de Vérité et méthode et qui est celle de la constitution ontologiquement langagière de l'être.

18. Kant réserve le concept de finitude dans sa première critique pour désigner le fait que la connaissance humaine est restreinte aux intuitions sensibles. Toutefois, nous croyons que la finitude est également, bien que de façon implicite, thématisée dans la troisième critique. L'épreuve consistant à porter un jugement de goût en l'absence de références universelles peut même constituer la manifestation par excellence de la finitude. Cette conception de la finitude s'étendra de "l'esthétique" à "l'ontologie» avec, entre autres, Heidegger et Derrida. 
d'emblée donné et où il incombe à la subjectivité seule de le déterminer. Le jugement esthétique de goût de type réfléchissant est celui pour lequel, contrairement au jugement déterminant, le particulier se trouve en face d'un vide laissé par un universel absent. Kant écrit :

La faculté de juger en général est la faculté qui consiste à penser le particulier comme compris sous l'universel. Si l'universel (la règle, le principe, la loi) est donné, alors la faculté de juger qui subsume sous celui-ci le particulier est déterminante (...). Si seul le particulier est donné, et si la faculté de juger doit trouver l'universel "qui lui correspond", elle est simplement réfléchissante ${ }^{19}$.

La situation selon laquelle la faculté de juger se retrouve en face d'un particulier qu'elle doit considérer en l'absence de références universelles et normatives est celle que nous qualifions d'état non-idéal de finitude. C'est l'état qui, confronté à l'absence de critères universels et donc au vide ou au néant doit statuer en recherchant un substitut compensatoire 20 pour pallier ce manque.

Kant, comme on le sait, résout la problématique du jugement réfléchissant dans le sensus communis comme «principe subjectif qui détermine seulement par sentiment et non par concept, bien que d'une manière universellement valable, ce qui plaît et ce qui déplaît ${ }^{21}$ ». Plus loin il ajoute :

Ainsi le sens commun, dont je donne comme exemple mon jugement de goût, lui conférant pour cette raison une valeur exemplaire, est une simple norme idéale.

19. Emmanuel Kant, Critique de la faculté de juger, Paris, Vrin, 1984, p. 27-28.

20. Pour une lecture de Kant qui n'en fait pas un théoricien des sciences, ni un ontologue, ni un protecteur de la métaphysique mais un précurseur du tournant esthétique de la philosophie, nous renvoyons à l'article du sceptique Odo Marquard "Kant und die Wende zur Ästhetik", Zeitschrift für Philosophische Forschung, Bd. 16 (1962), p. 231-243 et 363-374. Ce texte est également publié dans Odo Marquard, Aesthetica und Anaesthetica, München, Wien, Zürich, FERDINAND SCHÖNINGH, 1989.

21. Ibid., p. 78. 
(...) c'est que le principe, il est vrai seulement subjectif, mais cependant admis comme universellement subjectif (comme une ldée nécessaire à chacun), pourrait exiger, en ce qui concerne l'unanimité des différents sujets jugeant, une adhésion universelle tout de même qu'un principe objectif22.

Si nous reprenons ici le problème kantien d'une finitude qui se retrouve en face d'un vide d'universel, ce n'est pas pour défendre la solution proposée par Kant. Le problème trouve, admettons-le, une issue fort embarrassante dans l'idée d'un "principe admis comme universellement subjectif». La reprise de ce moment kantien poursuit plutôt les deux objectifs suivants qui consistent à montrer que :

1) la problématique du jugement réfléchissant liée à l'absence de singulier-universel dans le particulier-multiple peut être et a été transposée - d'abord par Heidegger - du champ de l'esthétique à celui de «l'ontologie fondamentale» (ce sera le thème de notre prochaine section);

2) Gadamer refuse d'assumer le problème naissant avec la modernité d'une finitude sans universel en s'empressant de désamorçer l'entreprise critique kantienne à l'endroit précis où celle-ci s'y confronte. La première section de Vérité et méthode portant sur l'expérience de l'art peut se lire comme un plaidoyer contre la difficulté kantienne au nom d'un retour à la conception d'un état de finitude sans doute plus rassurant et certainement plus classique que nous avons qualifié d'idéal. C'est ce geste que nous allons maintenant discuter.

Comme on le sait, Vérité et méthode souhaite dans sa première partie dépasser la sphère esthétique ${ }^{23}$ en neutralisant la "subjectivation de l'esthétique par la critique kantienne» 24 jusqu'à faire se coïncider jugement de goût et connaissance théorique ${ }^{25}$. Gadamer souhaite ainsi désubjectiver l'esthétique au profit d'une expérimentation cognitive de l'art. On pourrait bien sûr se demander pourquoi Gadamer rejette aussi caté-

22. Ibid., p. 79-80.

23. Gadamer, Vérité et méthode, Paris, p. 19.

24. Ibid., p. 59.

25. Ibid., p. 57. 
goriquement l'héritage de l'émancipation de la subjectivité de la tradition occidentale. L'herméneutique ne devrait-elle pas l'intégrer plutôt que de tout mettre en œuvre pour s'en détacher? Et deviendrait alors l'herméneutique de Gadamer en se confrontant sérieusement à l'éventualité de la disparition de l'idéal normatif de compréhension universel qu'elle postule?

II est bien vrai, comme le constate Gadamer, que la critique kantienne subjective l'esthétique en conférant au jugement de goût une universalité subjective comme sens commun ${ }^{26}$. II est bien vrai aussi que Kant discrédite "toute connaissance théorique autre que la science de la nature 27 „. II est également vrai, comme le remarque encore Gadamer, que l'esthétique kantienne "signifie à la fois rupture infligée à une tradition et introduction d'un nouveau développement ${ }^{28}$ ". Nouveau développement qui pourrait bien signifier la possibilité d'une contamination du champ de l'ontologie fondamentale par la problématique du jugement réfléchissant.

Pourquoi Gadamer se refuse-t-il à braver le «nouveau développement» de la pensée kantienne désubjectivant l'expérience esthétique? Qu'est-ce qui a pu motiver Gadamer à refuser l'intégration positive d'une problématique telle que celle du jugement réfléchissant kantien dans son herméneutique? ॥ semble que cela lui évite d'affronter l'état non-idéal de finitude émergeant à la modernité. Nous croyons que c'est le conservatisme philosophique de Gadamer symbolisé par son amour de la sculpture grecque «sans bouffissure et sans empâtement superflu» qui le pousse à rebrousser chemin vers la conception classique de la finitude définie par contraste avec un univers transcendant, normatif et imitable, univers qui s'incarne chez Gadamer dans l'idéal de compréhension universelle. Par ce geste, l'herméneutique de Gadamer esquive la problématique de la finitude non-idéale c'est-à-dire confrontée à l'absence de lois universelles.

28. Ibid., p. 57. C'est nous qui soulignons. 
Dans la prochaine section, nous tenterons de voir comment ce vide contourné par l'hermeuneutique philosophique a été pris en charge par deux figures de la pensée contemporaine de I'herméneutique philosophique.

\section{Heidegger et Derrida}

II faut bien sûr saluer les travaux de Gadamer en ce qu'ils reconnaissent en l'herméneutique une dimension dominante de la philosophie de notre époque. Toutefois, il semble que Gadamer réponde trop rapidement à la décisive question qui motive la crise actuelle de la pensée en réhabilitant la conception traditionnelle de la finitude définie par contraste avec une entité transcendantale sur laquelle elle se modèle idéalement. Les idéaux de pureté classiques ou néoclassiques de Gadamer le forcent à esquiver la possibilité d'une finitude imparfaite ou non-idéale qui risquerait de faire plonger son entreprise dans un trop affolant néant. Ce plongeon, le second Heidegger et Derrida l'ont effectué.

Comme nous l'avons souligné, la notion de finitude nonidéale vient questionner la possibilité de philosopher en l'absence d'une nature normative, imitable et distributrice de lois. Les pensées du second Heidegger et de Derrida ont manié ce type de finitude non-idéal, lointain écho du jugement réfléchissant kantien, en se lançant le défi de philosopher sur le néant.

On peut quand même reconnaître chez Heidegger un questionnement de nature similaire à celui auquel s'est heurté le jugement réfléchissant kantien. Le projet du second Heidegger peut se lire comme une tentative de penser fondamentalement en l'absence d'une nature distributrice de règles. C'est dans cette optique que peut être entendue l'identité déclarée de l'être et du néant dans l'affirmation : «L'essence de l'être même comporte dès l'origine le néant 29 ".

Présente dans tout le parcours du second Heidegger, cette unité rend dès lors difficile le projet d'élaborer une science de

29. Martin Heidegger, Questions I et II, Paris Gamillard, 1968, p. 63. Voir aussi Martin Heidegger, Kant et le problème de la métaphysique, Paris, Gamillard, 1965, p. 282. 
l'interprétation qui ne soit pas elle-même interprétation. Dans son Entretien de la parole entre un Japonais et un qui demande (1953-1954), Heidegger revient sur le concept d'herméneutique de Être et temps et affirme maintenant délaisser le concept "pour laisser [son] chemin de pensée sans nom ${ }^{30}$ ". Après avoir affirmé l'existence d'un site qui ne soit plus métaphysique ${ }^{31}$, Heidegger refuse désormais de reprendre conceptuellement la thématique du cercle herméneutique et écrit «ll n'y a jamais un parler sur la parole 32 ».

La prise en charge par Heidegger du néant et de l'état nécessairement non-idéal de finitude de celui qui souhaite sérieusement penser ce néant l'amène à adopter une attitude méditative. C'est dans cette attitude méditative que Heidegger affirme «manquer le mystère est l'errer ${ }^{33}$ ", qu'il nous invitera à faire «un saut [Satz] qui part de l'être comme fond de l'étant pour sauter dans l'abîme, dans le sans-fond (Abgrund) ${ }^{34}$ ", qu'il s'abandonnera au «ll y a» (Es gibt) être ${ }^{35}$, qu'il pensera l'être comme Ereignis ${ }^{36}$ et qu'il expérimentera l'A$A \eta \theta \varepsilon \iota \alpha$, l'état de non-retrait, comme Lichtung, clairière de la présence et de l'absence dans la liberté de l'Ouvert ${ }^{37}$.

Cette énumération rapide de différents moments de la pensée du second Heidegger devrait faire sentir la persévérance avec laquelle celui-ci a tenté de jouer avec une nouvelle notion de finitude qui ne se définisse plus en regard d'une normativité externe et imitable. Option classique, comme on l'a assez vu, reprise par Gadamer lorsqu'il fixe sa conception de la finitude par contraste avec un modèle de compréhension universelle.

Derrida, comme Heidegger, choisit d'explorer la finitude seule et sans normes en la confrontant à sa façon au néant.

30. Martin Heidegger, Acheminement vers la parole, Paris, Gamillard, 1976,

p. 114.

31. Ibid., p. 128.

32. Ibid., p. 136. Voir aussi formulation similaire p. 137.

33. Martin Heidegger, Questions I et II, Paris, Gallimard, 1968, p. 186.

34. Ibid., p. 273.

35. Martin Heidegger, Questions III et IV, Paris, Gallimard, 1976, p. 215.

36. Ibid., p. 221.

37. Ibid., p. 297-299. 
Défi qu'il relève par le biais d'une critique du phonocentrisme (ou phallogocentrisme) de la métaphysique qui s'accorde de façon illégitime, selon Derrida, le pouvoir de nommer des oppositions conceptuelles et d'en hiérarchiser les termes. Cette critique prend forme dans une déconstruction systématique de ces régimes oppositionnels. La déconstruction vient ainsi hanter la métaphysique de l'intérieur en subvertissant les oppositions hiérarchiques telles que sens/non-sens, vrai/faux, intelligible/sensible, idéalité/déchéance, parfait/imparfait, finitude/plénitude... qui la gouvernent.

Contre la prétention à l'absolu d'une objectivité et l'autonomie d'un fondement ${ }^{38}$, la finitude exempte de normes externes mise en scène par Derrida se qualifie par un recommencement indéfini du mouvement vers l'originaire ${ }^{39}$. Fidèle à cette conception de la finitude sans normes imitables qui lui fassent front, Derrida use de néologismes ou "redéfinit» des concepts en leur supprimant toute connotation "phoniquement» ou "logiquement» significative. Derrida se fait encore plus pervers en s'amusant à raturer le sens des "concepts-clés» de son entreprise au fur et à mesure qu'il les écrit. C'est ainsi que la "différance», la «trace» et le "supplément» sont tous déclarés synonymes par Derrida. II écrit : "La trace (pure) est la différance 40 ", et ajoute plus loin : "supplément, autre nom de la différance 41 ». Synonymes, certes ces «concepts-clés» le sont tous et veulent exprimer ce qui est impossible à dire, l'indicible, l'impropre, l'absence de nature distributrice de lois qui seraient assimilables par la finitude.

Déjà trop doctrinale, l'instauration d'une herméneutique philosophique pose donc problème au second Heidegger de même qu'à Derrida. Dans les deux cas, l'enjeu de la pensée consiste à questionner l'efficience de la métaphysique à notre époque.

38. Jacques Derrida, Le problème de la genèse dans la philosophie de Husserl,

Paris, P.U.F., 1990, p. 2.

39. Ibid., p. 257.

40. Jacques Derrida, De la grammatologie, Paris, Minuit, 1967, p. 92.

41. Ibid., p. 215. 
Au cours de ce travail, nous avons voulu montrer que l'herméneutique philosophique récupère un concept classique et un idéal de finitude en définissant celle-ci par contraste avec la plénitude transcendantale qu'est la compréhension universelle. Or, finitude et compréhension universelle, à l'encontre de ce que Gadamer en dit, ne s'opposent pas totalement l'une à l'autre dans son herméneutique. Nous avons indiqué l'existence d'un passage entre les "deux mondes" dans le fait que l'idéalité de la finitude l'autorise à imiter la compréhension universelle. Tout se passant comme si la compréhension universelle distribuait des règles qui s'incarnent dans le dialogue pour en favoriser la bonne marche vers le sens et une vérité grandissante. Nous avons vu cette dynamique à l'œuvre dans Vérité et méthode où Gadamer en vient à abolir herméneutiquement la frontière entre les mots et «l'en-soi».

Nous avons critiqué cette reprise antique et revisitée d'un état idéal de finitude par l'herméneutique de Gadamer en tant qu'elle refuse de se confronter aux avancées de la modernité. En posant l'existence d'un modèle de compréhension universelle, l'herméneutique philosophique tourne le dos à la possibilité qu'une finitude doive philosopher en l'absence de normes transcendantales. L'herméneutique philosophique occulte donc la possibilité qu'une finitude puisse ne plus avoir rien d'autre à imiter que ce qu'elle se donne elle-même. Contournant volontairement cette éventualité dans la première grande section de Vérité et méthode Gadamer se détourne du problème qui accapare tout un pan de notre tradition et qui consiste à penser l'absence d'une nature distributrice de lois, c'està-dire la présence du néant, dans un état de finitude que nous avons qualifié de non-idéal.

C'est donc dire que l'herméneutique de Gadamer, au nom de valeurs néoclassiques, demeure trop distante vis-à-vis de la crise actuelle de la philosophie et mesure mal l'importance de son enjeu principal qui est la remise en question de son appar- 
tenance au mode métaphysique de philosopher 42 . II nous semble donc que l'herméneutique de Gadamer contourne à trop grands traits la crise de notre époque.

De l'autre côté, le second Heidegger et Derrida se sont bien livrés à la tâche de penser avec le néant et l'absence de plénitude. Toutefois, il semble que ces projets se heurtent à la difficulté de devoir parler de l'absence sans la trahir.

En outre, nous croyons qu'une rencontre profitable demeure possible entre l'herméneutique philosophique et une pensée sensible aux difficultés de subsumer la philosophie sous un quelconque emblème ou de rendre en elle effectifs de quelconques idéaux. Cette rencontre pourrait avoir lieu sur les bases de la distinction établie entre un état idéal de finitude et un état dit non-idéal de finitude de même que par le biais d'un retour aux réflexions heideggériennes autour de la liberté de pensée comme Ouverture. En ce lieu où seraient positivement accueillis la crise de la philosophie et la volonté de lui donner sens nous pourrions espérer voir surgir de nouvelles possibilités pour la pensée. L'une d'elle pourrait être de ne plus sentir la nécessité d'user du terme même d'herméneutique pour dire l'expérience philosophique.

\section{Alain Beaulieu \\ Université des Sciences Humaines de Strasbourg}

42. Dans Vérité et méthode, Gadamer demeure discret quant à la relation qu'entretiennent son herméneutique et la métaphysique. Les quelques indices de ce rapport qu'on y retrouve nous apprennent que Gadamer, fidèle à Heidegger, entend la métaphysique classique au sens d'une ontologie du Vorhandenes (sous-la-main) (p. 480) où l'être est conçu comme présence. II écrit à ce sujet : "ll faut se tenir à l'abri du préjugé en faveur de l'ontologie du Vorhandenes et aussi à l'abri du concept d'objectivité" (p. 481). Toutefois, l'argumentation autour du Vorhandenes qui sert à Heidegger à légitimer un dépassement de la métaphysique ne poursuit pas le même but chez Gadamer. Celui-ci approche finalement la question de son rapport à la métaphysique au terme de Vérité et méthode où il affirme : "lci également, nous pouvons partir de la tradition métaphysique, ce qui ne nous dispensera pas d'avoir à nous demander ce qui en elle demeure valable pour l'expérience herméneutique". (p. 512) Programme tardif dans l'économie de Vérité et méthode (4 pages avant la fin!) qui demeure en plan avec la fin du livre. Or, nous avons montré que la finitude de l'herméneutique philosophique est celle de la métaphysique. 